CONGENITAL HEART DISEASE

\title{
Infective endocarditis in congenital heart disease: Japanese national collaboration study
}

\author{
K Niwa, M Nakazawa, S Tateno, M Yoshinaga, M Terai
}

Heart 2005;91:795-800. doi: 10.1136/hrt.2004.043323

Objective: To provide pure cohorts of paediatric and adult patients with congenital heart disease (CHD) and infective endocarditis (IE) for making future guidelines.

Design: Japanese nationwide survey.

Setting: 66 Japanese institutions.

Patients: 170 children, mean (SD) age 7.4 (5.7) years (range 14 days to 17 years), and 69 adults, age 32.5 (14.1) years (range 18-69) who developed IE between 1997 and 2001 (one in 240 admissions with CHD).

Main outcome measures: Clinical presentation of IE.

Results: 119 patients including 88 with cyanotic CHD had previous cardiac surgery. Procedures preceding IE were dental (12\%) followed by cardiovascular surgery ( $8 \%$ ). Sites of infection were left sided in $46 \%$ and right sided in $51 \%$. Vegetation with diameter of $11 \mathrm{~mm}$ was documented in 151 (63\%). Frequent complications were embolic events (stroke $11 \%$, other emboli $20 \%$ ) and cardiac failure (23\%). The most

See end of article for authors' affiliations

.....................

Correspondence to:

Dr K Niwa, Department of

Paediatrics, Chiba

Cardiovascular Centre,

575 Tsurumai, Ichihara

Chiba 290-0512 Japan;

kniwa@aol.com

Accepted 14 August 2004

.................... common microorganisms were streptococci $(50 \%)$ and staphylococci $(37 \%)$ with methicillin resistant Staphylococcus aureus in 7.5\%. Empirical treatments were penicillins (alone or with other antibiotics 57\%) followed by cephems (22\%) and vancomycin (11\%). Surgery during active IE was common (26\%), with vegetation $(45 \%)$ and heart failure $(29 \%)$ as the most frequent indications. Mortality was $8.8 \%: 8.0 \%$ among patients who received medical treatment alone and $11.1 \%$ among those with active IE who underwent surgery. The causes of death $(n=21)$ were surgery (7), infection (7), cardiac failure (6), and renal failure (1).

Conclusions: Because of a recent increase in the incidence of IE and high mortality and complication rate, it is mandatory to establish well formulated recommendations for management of IE in paediatric and adult patients with CHD based on a large cohort. Results of this nationwide multicentre database should be helpful in establishing guidelines.
$\mathrm{P}$ ublished guidelines used worldwide for infective endocarditis (IE) $)^{1-6}$ address mainly adults with acquired cardiac disorders. Cardiologists have not had access to formulated guidelines for IE in paediatric and adult patients with congenital heart disease (CHD) based on data from a pure large cohort. Increasing numbers of patients with CHD have now reached adulthood ${ }^{7}$ and CHD constitutes the major substrate for IE in both children and adults. ${ }^{8-14}$ Because relatively few cases of CHD and IE are seen in any one institution, authors have tended to report the issue of IE by combining their clinical experience over several decades. ${ }^{9-12} 1516$ Accordingly, there is a dearth of practical and current data of IE from a large cohort of paediatric and adult patients with CHD. ${ }^{8}$

This study aimed at clarifying recent information on the clinical presentation of IE in a nationwide survey in Japan for the formulation of the guidelines in paediatric and adult patients with CHD.

\section{METHODS}

Information regarding the history, demographics, prophylaxis, diagnosis, and management of IE in 239 patients with a mean (SD) age of 14.7 (14.5) years (range 14 days to 69 years) from January 1997 to December 2001 was obtained from 66 institutional databases and analysed at the Chiba Cardiovascular Centre, Chiba, Japan. The 239 patients comprised 170 children (147 with CHD and 23 without apparent underlying heart disease), aged 7.4 (5.7) years (range 14 days to 17 years), and 69 adults with CHD, aged 32.5 (14.1) years (range 18-69 years). Admissions with CHD during the five year period were 50985 with a prevalence of IE of $0.42 \%$ (212 of 50985 or one in 240) (excluding two institutions with four patients with CHD in which the total number of admissions was unknown).

\section{Collection of data regarding IE}

The following data regarding IE were solicited.

- Demographic data: age, sex, diagnosis of CHD, previous cardiac surgery (repair or palliative), residual lesion after repair and history of recurrent IE; in patients with previous cardiac surgery, whether IE was perioperative (perioperative was defined as the onset of IE within six months after surgery)

- Prophylaxis: whether IE prophylaxis was undertaken; the procedures and conditions requiring IE prophylactic in terms of choice, dose, duration

- Bacteriology: the causative microorganism; whether antibiotics were administered before blood was cultured and, if blood was cultured, the number of cultures for each patient and whether the culture was positive or negative

- Echocardiographic findings: site and size of vegetation, enhanced intensity of endocardium, perforation of valvar leaflet, and periannular extension of abscess

Abbreviations: $C H D$, congenital heart disease; $I E$, infective endocarditis; MRSA, methicillin resistant Staphylococcus aureus; TOE, transoesophageal echocardiography 
- Pathological findings (necropsy and surgery): site and size of vegetation, periannular extension of abscess, prosthetic material infection, and systemic emboli

- Complications: whether complications were observed (complications were increased valvar regurgitation, cardiac failure, cerebral or systemic embolisation, arrhythmia, periannular extension of abscess, prosthetic valve dysfunction, glomerular nephritis or renal failure, and mycotic aneurysm)

- Management: choice, dose, and duration of antibiotics, and interval between diagnosis and initiation of antibiotics; whether complications, anticardiac failure regimens, antiarrhythmic medication, and neurosurgery for mycotic aneurysm were managed; and timing, indication, surgical method, and mortality of cardiac surgery after IE

- Mortality: whether the patient was alive or dead and cause of death; among patients who died, whether they were treated with drugs alone, underwent surgery during active IE (before a full course of antibiotics had been completed), or underwent surgery with healed IE (after a full course of antibiotics had been completed).

\section{Statistical analysis}

Data were expressed as mean (SD) with range, the number and percentage, or the number of answers as a percentage of total number of answers. When a question had no response or when an answer was unclear, those data were not counted. Available data from participating hospitals were analysed with StatView J-5.0 PPC (SAS Institute Inc, Cary, North Carolina, USA). Discrete variables were analysed by $\chi^{2}$ test. Continuous data were compared between subgroups by two sample $t$ test or the Wilcoxon rank sum test wherever appropriate. When $\mathrm{p}<0.05$, the data were defined as significant.

\section{RESULTS}

\section{Demographic data}

The male to female ratio was 143:96. Of the population of 239, 20 (8.4\%) were infants aged 5.1 (3.3) months (range 14 days to 10 months). Table 1 lists the diagnoses of CHD. No patients had rheumatic heart disease. Previous reparative or palliative cardiac surgery was undertaken in 119 patients with CHD ( 119 of 216 (55.1\%), 75 with reparative surgery and 44 with palliative surgery) including 88 with cyanotic CHD. A residual defect after repair was observed in 55 of 75 $(73.3 \%)$ patients. IE recurred in 21 of $229(9.2 \%)$ patients: once in 10, twice in two, three times in two, and four or more times in two. In 28 of $119(23.5 \%)$ patients with previous cardiac surgery, IE developed perioperatively.

\section{Prophylaxis}

In 78 of $234(33.3 \%)$ patients, conditions and procedures associated with IE were identified (table 2). In 22 of 78 $(28.2 \%)$, prophylactic antibiotic regimens were administered intravenously ( 14 patients) or orally (eight patients). In nine of 14 patients with intravenous prophylaxis, methicillin resistant Staphylococcus aureus (MRSA) $(\mathrm{n}=7)$ or Candida albicans $(\mathrm{n}=2$ ) was the causative microorganism. In eight patients with oral prophylaxis, streptococci were found in three and staphylococci in four. No patients used injected drugs.

\section{Site of infection}

The site of infection was identified (175 of 239 of patients $(73.2 \%)$ ) more often in paediatric patients (127 of 170 $(74.7 \%)$ ) than in adults (48 of $69(69.6 \%), p=0.1)$. Left sided IE was observed in 81 of $175(46.3 \%)$ patients: mitral valve, $44(25.1 \%)$; aortic valve, 26 (14.9\%); both the mitral and aortic valves, five (2.9\%); left atrium, two (1.1\%); left ventricular outflow tract, two (1.1\%); and ascending aorta, arch, or both, six (3.4\%). Right sided IE was observed in 89 of $175(50.9 \%)$ patients: tricuspid valve, 37 (21.1\%); ventricular septal defect, 19 (10.8\%); right ventricle, 12 (6.9\%); right ventricular outflow tract conduit, 10 (5.7\%); pulmonary valve, nine (5.1\%); pulmonary artery, four $(2.2 \%)$; left carotid vein, one $(0.5 \%)$; and atrial septal closure suture, one $(0.5 \%)$. IE in both right and left ventricles was observed in four $(2.3 \%)$ and Blalock-Taussig shunt in two (1.1\%). In adults, the prevalence of left sided IE (29 of $48(60.4 \%)$ ) was higher than that of right sided IE ( 21 of $52(40.4 \%)$. In 23 children without underlying structural heart disease, sites of infection were mitral valve, 15; aortic valve, five; both mitral and aortic valve, two; and tricuspid valve, one (IE due to central venous catheter insertion).

\section{Bacteriology}

Causative microorganisms were identified in 201 of 239 $(84.1 \%)$ patients (table 3 ).

\section{Diagnosis of IE}

Blood samples were cultured 3.5 (range 1-18) times for each patient. The mean (SD) interval between initial blood culturing and initiation of antibiotics was 90.8 (150) hours (range 0-720 hours).

Transoesophageal echocardiography (TOE) was performed in 30 of $228(13.2 \%)$ patients and 23 of them were adults. IE findings were positive in 167 of 235 (71.1\%) patients: vegetations, $145(61.7 \%)$; enhanced intensity of endocardium, 127 (54.0\%); perforations of valve leaflet, 17 (7.2\%); and periannular extension of abscess, 14 (6.0\%). Mean (SD) vegetation size was 11.1 (6.7) $\mathrm{mm}$ (range $1-39 \mathrm{~mm}$ ). Vegetation size in patients with systemic embolisation ( $\mathrm{n}=26)$ was $11.5(6.5) \mathrm{mm}$ (range $1-27 \mathrm{~mm})$, which was similar to the size in patients without systemic embolisation (10.9 (6.3) $\mathrm{mm}$ (range $1-39 \mathrm{~mm})$ ). In six patients, vegetations of $6 \mathrm{~mm}$ were identified by pathological examinations but were not found by echocardiographic study. Vegetation was detected in 39 of $67(58.2 \%)$ patients with a size of $11.1 \mathrm{~mm}$ in adults, which was similar to the size in paediatric patients ( 106 of $170(62.4 \%), 11.1 \mathrm{~mm}, \mathrm{p}=0.2$ ).

\section{Prosthetic material infection}

Prosthetic material was infected in 26 of 136 (19.1\%) patients: prosthetic valve, four; right ventricular outflow conduit, 10; right ventricular outflow patch, two; ventricular septal defect patch, six; Blalock-Taussig shunt, two; pulmonary artery banding, one; and ventriculoatrial shunt, one.

\section{Complications}

Complications were observed in 116 of 239 (48.5\%) patients after a mean (SD) of 29 (42) days (range 0-180 days) of illness (table 4). In 19 of 25 of patients with cerebral emboli, IE was associated with $S$ aureus. In 37 of 48 of patients with embolisation, sites of vegetation were identified as the mitral valve in 10, aortic valve in seven, tricuspid valve in six, pulmonary valve in three, right ventricular outflow tract in four, atrial and mitral valves in one, conduit in one, right ventricle and mitral valve in one, pulmonary artery in one, left atrium in one, atrial, mitral, and tricuspid valves in one, and aortic arch in one. Thirteen patients received antiarrhythmic medication and a pacemaker was implanted in one patient with complete atrioventricular block. Patients with periannular extension of the abscess did not manifest heart block. Medication for cardiac failure was administered to 57 patients. One child underwent neurosurgery for a mycotic aneurysm. 
Table 1 Diagnoses of underlying congenital heart disease

\begin{tabular}{|c|c|c|c|c|}
\hline Diagnosis & Total $(n=216)$ & $\begin{array}{l}\text { Non-operated } \\
(\mathrm{n}=97)\end{array}$ & Repaired ( $n=75$ ) & Palliated $(n=44)$ \\
\hline VSD, VSD + ASD or PDA & $81(37.5 \%)$ & 65 & VSD closure: 16 & 0 \\
\hline $\begin{array}{l}\text { Tetralogy of Fallot with/without } \\
\text { pulmonary atresia }\end{array}$ & $39(18.1 \%)^{*}$ & 3 & Rastelli: 11, ICR: 8 & Unifocalisation: 4, AP shunt: 13 \\
\hline Single ventricle (heterotaxia) & $19(8.8 \%)$ & 3 & TCPC: 2, Fontan: 2 & AP shunt: $10, P A B: 2$ \\
\hline Double outlet right ventricle & $16(7.4 \%)$ & 0 & Rastelli: 2, Fontan: 3, ICR: 3 & AP shunt: 6, PAB: 2 \\
\hline Mitral stenosis/regurgitation & $15(6.9 \%)$ & 12 & MVR: 1, MVP: 2 & \\
\hline $\begin{array}{l}\text { Aortic stenosis/regurgitation } \\
\text { (bicuspid aortic valve) }\end{array}$ & $11(5.1 \%)$ & 7 & Ross: 1, AVR: 1, AVP: 2 & \\
\hline Complete TGA & $8(3.7 \%)$ & 0 & Rastelli: 5, Jatane: 1 & AP shunt: 2 \\
\hline COA, COA VSD & $6(2.8 \%)$ & 0 & VSD closure: 4 & SCLF: 2 \\
\hline AVSD & $6(2.8 \%)$ & 2 & AVSD closure, MVP: 3 & AP shunt: 1 \\
\hline Corrected TGA & $5(2.3 \%)$ & 0 & Rastelli: 2; TVR: 1, VSD closure: 1 & AP shunt: 1 \\
\hline ASD & $2(0.9 \%)$ & 1 & Closure: 1 & \\
\hline PDA & $2(0.9 \%)$ & 2 & & \\
\hline Truncus arteriosus communis & $2(0.9 \%)$ & 0 & Rastelli: 2 & \\
\hline Valsalva aneurysm & $2(0.9 \%)$ & 2 & & \\
\hline Ebstein's disease & $1(0.5 \%)$ & 0 & TVP: 1 & \\
\hline HLHS & $1(0.5 \%)$ & 0 & & Norwood: 1 \\
\hline \multicolumn{5}{|c|}{$\begin{array}{l}\text { *Tetralogy of Fallot and pulmonary atresia: } 22 \text {. } \\
\text { AP, aortopulmonary; ASD, atrial septal defect; AVP, aortic valvoplasty; AVR, aortic valve replacement; AVSD, atrioventricular septal defect; COA, coarctation of } \\
\text { the aorta; HLHS, hypoplastic left heart syndrome; ICR, intracardiac repair; MVP, mitral valvoplasty; MVR, mitral valve replacement; PAB, pulmonary artery } \\
\text { banding; PDA, patent ductus arteriosus; SCLF, subclavian flap; TCPC, total cavopulmonary connection; TGA, transposition of the great arteries; TVR, tricuspid } \\
\text { valve repair; VSD, ventricular septal defect. }\end{array}$} \\
\hline
\end{tabular}

\section{Antimicrobial treatment}

Table 5 shows empirical antimicrobial treatment $(n=235)$. Antibiotics were started at 18.5 (33.3) days (range 0-240 days) of illness, which was within seven days in 104 of 203 (51.2\%) patients. The duration of antibiotics administration was 29.7 (16) days (range 1-70 days). Antibiotics and antifungal agents were ( $\mathrm{n}=420$ in 235 patients) penicillins in $147(63 \%)$, cephems in $81(35 \%)$, aminoglycosides in 84 $(36 \%)$, carbapenems in $43(18 \%)$, vancomycin in $29(12 \%)$, oxacephems in nine $(4 \%)$, tetracyclines in nine $(4 \%)$, fosfomycin in seven (3\%), fluconazole in five $(2.1 \%)$, clindamycin in four $(2 \%)$, and chloramphenicol in one $(0.4 \%)$.

\section{Cardiac surgery}

Cardiovascular surgery for IE was performed in 103 of 239 (43.1\%) patients. This was during active IE (full course of antibiotics had not been completed) in 63 of $239(26.4 \%)$ at 37.2 (34.8) days (range 0-180 days) of illness and in healed IE (full course of antibiotics had been completed) in 40 of 239 (16.7\%) patients at 301 (456) days (range 30 days to 49 months) of illness. In 12 of 26 of patients with prosthetic material infection, cardiac surgery was undertaken during active IE. Table 6 lists indications for cardiac surgery.

Table 2 Conditions and procedures associated with infective endocarditis

\begin{tabular}{ll}
\hline Conditions and procedures & Number $(\mathbf{n}=\mathbf{7 8})$ \\
\hline Invasive dental procedures & $29(37.2 \%)$ \\
Cardiovascular surgery & $20(25.6 \%)$ \\
Pneumonia & $11(14.1 \%)$ \\
Invasive otolaryngeal procedures & $5(6.4 \%)$ \\
Unexpected trauma & $4(5.1 \%)$ \\
Atopic dermatitis & $4(5.1 \%)$ \\
Central venous catheter & $2(2.6 \%)$ \\
Catheter intervention & $1(1.3 \%)$ \\
Meningitis & $1(1.3 \%)$ \\
Piercings & $1(1.3 \%)$ \\
\hline
\end{tabular}

\section{Mortality}

Total mortality was 21 of $239(8.8 \%)$. Fourteen of $176(8.0 \%)$ patients who received medical treatment alone $(\mathrm{n}=176)$ died. None of $40(0 \%)$ patients who had surgery $(\mathrm{n}=40)$ after IE had healed died. Seven of $63(11.1 \%)$ patients who had surgery $(n=63)$ during active IE died, which was similar to mortality with medical treatment alone $(\mathrm{p}=0.2)$.

The mortality among paediatric patients ( 16 of $170(9.4 \%)$ ) was similar to that of adults (five of $69(7.2 \%), p=0.6$ ). Three of the 12 patients with prosthetic material infection died: one with medical treatment alone and two others who underwent surgery during acute IE.

\section{Causes of death}

The causes of death in 21 patients were surgery in seven, cardiac failure in six (including two with cerebral embolisation), IE unresponsive to antimicrobials in six, pneumonia in one, and renal failure in one.

\section{DISCUSSION}

Clinical features of IE in 239 patients-170 paediatric and 69 adults-with CHD are established. The incidence of IE in

\begin{tabular}{|ll|}
\hline Table 3 & Causative microorganisms \\
\hline Organism & Number $(\mathbf{n}=201)^{*}$ \\
\hline Streptococcus species & $100(49.8 \%)$ \\
$\alpha$ Streptococcus & $87(43.3 \%)$ \\
Pneumonia & $11(5.5 \%)$ \\
$\beta$ Streptococcus & $2(1.0 \%)$ \\
Staphylococcus species & $74(36.8 \%)$ \\
S aureus & $64(31.8 \%)$ \\
MRSA & $15(7.5 \%)$ \\
S epidermidis & $10(5.0 \%)$ \\
MRSE & $4(2.0 \%)$ \\
Haemophilus species & $9(4.5 \%)$ \\
Candida species & $5(2.5 \%)$ \\
Pseudomonas & $4(2 \%)$ \\
Others† & $9(4.5 \%)$ \\
\hline *Unknown causative microorganism: $38 ; \dagger$ Granulicatella \\
species 3, Micrococcus species 1, Moraxella species 1, \\
Gamella species 1, enterococci 1, bacillus 1, anaerobic \\
species 1. \\
MRSA, methicillin resistant Staphylococcus aureus; MRSE, \\
methicillin resistant Staphylococcus epidermidis. \\
\hline
\end{tabular}




\begin{tabular}{|ll|}
\hline Table 4 Complications & \\
\hline Complications & $\begin{array}{l}\text { Number ( } \mathbf{n}=116, \\
\text { multiple answers) }\end{array}$ \\
\hline Increased valvar regurgitation & $73(30.5 \%)$ \\
Cardiac failure & $56(23.4)$ \\
Systemic emboli & $48(20.1)$ \\
Stroke & $25(10.5 \%)$ \\
Systemic abscess formation & $12(5 \%)$ \\
Arrhythmia development & $13(5.4 \%)$ \\
Periannular extension of abscess & $11(4.6 \%)$ \\
Intracranial haemorrhage & $7(2.9 \%)$ \\
Prosthetic valve dysfunction & $4(1.7 \%)$ \\
Glomerulonephritis/renal failure & $4(1.7 \%)$ \\
Mycotic aneurysm & $3(1.3 \%)$ \\
\hline
\end{tabular}

patients with CHD is increasing, especially in infants and adults with previous cardiac surgery. Despite recent developments in prevention and diagnosis of IE and medical and surgical management of acute IE, complication rates and mortality are still too high. Because of the high mortality caused by IE among patients with CHD (8.8\%) it is mandatory to establish formulated recommendations for the medical and surgical management of paediatric patients and adults with IE and CHD based on a large database. Results of this nationwide multicentre database should be helpful in achieving this goal.

\section{Demographic data}

The prevalence of IE in patients with CHD has been increasing. ${ }^{8-15}$ Morris $^{14}$ reported that the highest risk for IE was found in children who have had repair or palliation of cyanotic CHD. In this study, the prevalence of IE, especially in infants and adults with CHD and repaired or palliated cyanotic CHD is high. An increased prevalence of perioperative IE in infants reflects the recent strategy of early cardiovascular repair in neonates and infants with complex CHD. Indeed, the proportion of patients with previous surgery is high (about $50 \%$ in published reports ${ }^{216-18}$ and $55 \%$ in our study). IE in adults with CHD has been increasing along with the increasing number of patients with CHD reaching adulthood..$^{12-14}$

\section{Diagnosis of CHD and cardiac conditions associated with IE}

Ventricular septal defect, patent ductus arteriosus, aortic valve abnormalities, and tetralogy of Fallot are reported to be common underlying conditions for IE. ${ }^{2} 9101519$ In this study, ventricular septal defect was the most frequent underlying condition with patent ductus arteriosus and aortic abnormalities lower than in previous reports. ${ }^{9} 10$ Instead, cyanotic CHD, such as tetralogy of Fallot, single ventricle with or without heterotaxia, double outlet right ventricle, and transposition of the great arteries $(38 \%)$ were more frequent. The most common lesion to develop IE after surgery is reportedly tetralogy of Fallot or an aortopulmonary shunt. ${ }^{2}$ In this study, aortopulmonary shunt, extraconduit repair, and prosthetic material were frequent lesions.

Corrective surgery usually eliminates the risk for IE in patients with left to right shunts such as ventricular septal defect or persistent ductus arteriosus. ${ }^{2}{ }^{16}$ In this study we observed residual defect in $82 \%$ of 75 patients with repaired defects. Close observation of residual lesions in patients after repair is therefore very important for prevention of IE. The risk of IE in patients with repaired defects with aortic stenosis is high regardless of residual haemodynamic defect. ${ }^{15} 16$ In this study, four of 11 patients with previous aortic valve surgery developed IE.
Table 5 Empirical antimicrobial treatment

\begin{tabular}{ll}
\hline Antibiotics & Number (n=235) \\
\hline Penicillins alone & $54(23 \%)$ \\
Penicillins plus aminoglycosides & $50(21 \%)$ \\
Penicillins plus others* & $30(13 \%)$ \\
Cephems alone & $29(12 \%)$ \\
Cephems plus aminoglycosides & $8(3.4 \%)$ \\
Cephems plus others† & $15(6 \%)$ \\
Carbapenems alone & $9(4 \%)$ \\
Carbapenems plus others $\ddagger$ & $7(3 \%)$ \\
Vancomycin alone & $4(1.7 \%)$ \\
Vancomycin plus carbapenems & $6(2.6 \%)$ \\
Vancomycin plus others§ & $17(7 \%)$ \\
Oxacephems & $4(1.7 \%)$ \\
Antifungal agents (fluconazole) & $2(0.8 \%)$ \\
\hline *Cephems, aminoglycosides, carbapenems, clindamycin, or \\
tetracyclines; †carbapenems, fosfomycin, aminoglycosides, or \\
tetracyclines; łaminoglycosides, oxacephems, fosfomycin, clindamycin, \\
or tetracyclines; §aminoglycosides, cephems, tetracyclines, or \\
carbapenems.
\end{tabular}

It is reported that drug misuse is often associated with IE in adults in the USA and Europe, ${ }^{1}$ but there were no patients associated with drug abuse in this study or in a recent nationwide survey of IE in adults in Japan. ${ }^{13}$

\section{Prophylaxis}

Significant bacteraemia is defined by the American Heart Association as organisms commonly associated with IE and attributable to identifiable procedures such as dental, oral, and respiratory tract procedures. ${ }^{1}$ In our study, the most frequent procedure associated with IE was dental followed by cardiac surgery. Otolaryngeal procedures, pneumonia, and atopic dermatitis were also important predisposing causes or conditions for IE in patients with CHD. ${ }^{13}$

Prophylaxis is recommended for patients who undergo open heart surgery and should be aimed primarily against $S$ aureus and coagulase negative staphylococci, for which a first generation cephalosporin or vancomycin is thought to be a reasonable choice. ${ }^{1}$. In this study, seven of 11 patients developed perioperative IE associated with MRSA or fungus resistant to the first choice antibiotics, cephalosporins. Most surgeons, as reported recently, used cephalosporins as first choice perioperative prophylactic agents. ${ }^{8}$ The choice of perioperative antibiotics should be tailored according to patient conditions and institutional circumstances.

\section{Site of IE}

In children with CHD, right sided IE is reported to be more frequent than left sided, but in adults mainly with acquired heart disease, left sided IE is more prevalent. ${ }^{20}$ In adults with CHD in this study, left sided IE was more frequent than right

Table 6 Indications of surgery in active infective endocarditis

\begin{tabular}{ll}
\hline Indication & $\begin{array}{l}\text { Number (n=63, } \\
\text { multiple answers) }\end{array}$ \\
\hline $\begin{array}{l}\text { Vegetation: possible future development of } \\
\text { systemic embolisation/status after systemic } \\
\text { embolisation }\end{array}$ & $37(52.4 \%)$ \\
$\begin{array}{l}\text { Cardiac failure unresponsive to medical } \\
\text { treatment }\end{array}$ & $20(31.7 \%)$ \\
$\begin{array}{l}\text { Prosthetic material infection } \\
\text { Periannular extension of abscess }\end{array}$ & $14(20.3 \%)$ \\
Persistent bacteraemia despite appropriate & $11(17.5 \%)$ \\
medical treatment & $5(7.9 \%)$ \\
Arrhythmia & $2(2.9 \%)$ \\
Others & $4(5.8 \%)$ \\
\hline
\end{tabular}


sided IE. Right sided lesions susceptible to IE were common in CHD. About $10 \%$ of reported paediatric patients and IE have no underlying structural heart disease and the site of IE was usually the aortic or mitral valve associated with $S$ aureus bacteraemia. $^{29}$ In this study, 23 children $(13.8 \%$ of the paediatric patients) developed IE without structural heart disease and the site of IE was a left sided cardiac lesion in all except one. The results were similar to previous reports, ${ }^{29}$ but about half of the cases were associated with streptococcal bacteraemia.

\section{Microorganism}

The organisms that cause IE in children most often are Gram positive cocci $(32-43 \%) .{ }^{19}{ }^{10}$ With the increasing frequency of cardiac surgery in patients with complex CHD in which prosthetic materials are used, the incidence of infection with staphylococci, Gram negative bacilli, and fungi has been increasing. ${ }^{20}{ }^{21}$ In this study, Gram positive cocci, especially Streptococcus species (50\%) and Staphylococcus species (37\%), were the most frequent microorganism followed by Haemophilus (4.5\%) and Candida species (2.5\%). In Staphylococcus species, $S$ aureus (with 24\% MRSA) was the most frequent microorganism.

\section{Echocardiography}

Transthoracic echocardiography is more likely to identify vegetations in patients with normal anatomy or isolated valvar pathology than in complex cyanotic CHD as a result of interference in complex cyanotic CHD by arterial grafts, conduits, and valves. ${ }^{2819} 22$ The low detection rate $(62 \%)$ of vegetation by echocardiography in both paediatric and adult patients in this study may be attributable to our higher prevalence of post-surgical patients with complex CHD with prosthetic materials.

In this study, TOE was used in only a small number of patients, especially in adults. TOE is superior to transthoracic echocardiography in detecting vegetations in the adult cohort. ${ }^{3}$ However, this was not a study of the usefulness of TOE in paediatric cohorts. Paediatric patients usually have a much better echo window than do adults. ${ }^{89}$

\section{Blood culture}

Two to three sets of blood cultures over a 24 hour period is thought to be adequate in most cases in children with $\mathrm{IE}^{23}$ and blood samples were cultured 3.5 times for each patient in this study. The positive rate of pathogens grown in blood culture was reported to be $68-98 \%^{21}$ and the rate dropped to $60 \%$ when antibiotics were used before blood culturing. ${ }^{24}$ In this study, blood cultures were positive for pathogens in $86 \%$ of the patients despite the high incidence of prior administration of antibiotics (69\% of the patients; most of these were administered orally) before blood culturing.

\section{Complications}

Cardiac failure, usually accompanied by valve regurgitation, is one of the most important predictive factors for mortality of IE. ${ }^{21}$ Prosthetic valve IE is common and usually results in valve replacement in adults. ${ }^{3}$ Extension of IE beyond the valve annulus predicts higher mortality with more frequent development of cardiac failure and the need for cardiac surgery. ${ }^{3}$ In this study, cardiac failure worsened because of IE in $21 \%$ of patients. Periannular extension of the abscess was observed in only $5 \%$ and prosthetic valve IE was observed in $1.7 \%$, which is consistent with previous reports on paediatric patients with IE. ${ }^{25}$ Neurological signs and symptoms are often associated with $S$ aureus infection and are reported in $20 \%$ of children with IE. ${ }^{18}$ In this study, cerebral emboli were observed in $11 \%$ of the patients and $76 \%$ were associated with $S$ aureus infection.
Because the likelihood of cure with antibiotics alone is decreased, surgical intervention is often required. A potentially life threatening complication is the development of IE in a surgically created shunt or conduit in patients with complex CHD. In this study, prosthetic material was infected in 26 of 120 patients with previous surgery $(22 \%)$ and 12 required urgent cardiac surgery that resulted in three deaths.

Vegetation of $10 \mathrm{~mm}$ or greater is a predictive sign of embolisation, especially a mitral valve vegetation. ${ }^{36}$ In this study, the size of vegetation was $11 \mathrm{~mm}$ and systemic embolisation was observed in 20\% of the patients: $55 \%$ of the embolisations were cerebral. Vegetation size in patients with embolisation $(n=26)$ in this study was 11.5 (6.5) $\mathrm{mm}$; a similar number of patients did not have embolisation. The most frequent site of vegetation that resulted in embolisation is reported to be the mitral valve but in this study in patients with CHD, the aortic and tricuspid valves were also frequent foci for embolisation. Mycotic aneurysms are uncommon complications of IE and are very rare, especially in children. ${ }^{327}$ In this study, mycotic aneurysm was observed in two adults, and one child required neurosurgery. The development of new atrioventricular or bundle branch block is a frequent sequela of periannular extension of the abscess in adults. ${ }^{3}$ In this study, no patients with conduction defects had periannular extension of the abscess.

\section{Medical management of IE}

For empirical infection, penicillins alone or penicillins combined with aminoglycosides are widely recommended. ${ }^{3-6}$ In this study, penicillins (alone or combined with aminoglycosides) were most often used antibiotics for this purpose (57\%), but about $20 \%$ of the physicians preferred cephems and $12 \%$ preferred vancomycin (or a combination of others).

\section{Mortality and cardiac surgery in patients with active IE}

The prognosis of children with IE depends on many factors, such as severity of underlying cardiac lesion, presence of prosthetic materials, type of infecting organism, and duration of illness before diagnosis. Mortality among paediatric patients with CHD and IE is reported to be lower than among adults with acquired heart disease. ${ }^{21}$ The recently reported mortality among paediatric patients with IE is about $10 \%,{ }^{19}{ }^{21}$ which is similar to our mortality $(9.4 \%)$ and higher than that of adults with CHD (7.2\%).

The incidence of reinfection of newly implanted valves in patients with active IE has been estimated at $2-3 \%$ in adult patients, ${ }^{3}$ and surgery as an adjunct to medical treatment was recognised as more effective than medical treatment alone. ${ }^{36-32}$ In this study, $26 \%$ of the patients underwent cardiac surgery during active IE with no recurrence but with higher mortality than with medical treatment alone. The risk factors for death were not measured in this study; however, this relatively higher mortality among patients with surgery in the acute phase may be attributable to differences in factors such as the timing of the operation, severity of cardiac disease, rate of complication, or differences in microorganisms between these two subgroups.

Indications for surgery in active IE are uncontrollable cardiac failure or infection, an episode of embolisation, fungal IE, perivalvar abscess, and prosthetic materials, such as prosthetic valve, conduit, aortopulmonary bypass graft, or patch infection. ${ }^{29-32}$ The most frequent indication for surgery in this study was a vegetation likely to develop systemic embolisation or the status after systemic embolisation and heart failure. The current practice of cardiac surgery for CHD is the frequent use of prosthetic material in a variety of cardiac locations. A high mortality has been reported among 
patients with IE with infected prosthetic materials ${ }^{30}$ requiring replacement. ${ }^{10}{ }^{19}$ In this study, infected prosthetic material was surgically removed from 17 patients with a resultant mortality of $12 \%$.

Because of the recent increase in the incidence and the high mortality from IE $(8.8 \%)$, it is mandatory to establish formulated recommendations for the medical and surgical management of IE in paediatric and adult patients with CHD based on a large database.

\section{Limitations}

Most cardiologists in Japan use the Duke criteria for diagnosing IE, but some in the participating institutions had no uniform criteria ${ }^{8}$ and the diagnosis of IE in the reported cases was not validated. Our patients had a large age range from infants and children to adults with CHD. Many cardiologists participated in this study; therefore, these factors may cause a bias in the analysis of the clinical profile of IE in Japanese patients with CHD.

\section{Conclusions}

Precise features of IE in 239 paediatric and adults patients with CHD are clarified. An increasing number of patients, especially infants and adults with cyanotic CHD and previous surgery, are developing IE. Despite recent advances in diagnosis and treatment, mortality and the rate of complications, such as cardiac failure and systemic embolisation, are high. To reduce mortality and morbidity in patients with CHD and IE, it is necessary to establish guidelines for IE prevention and management based on a large cohort of patients. The nationwide multicentre database was designed to meet this need, and the results should be helpful in establishing guidelines for IE in CHD.

\section{ACKNOWLEDGEMENTS}

We gratefully acknowledge the assistance of $\mathrm{N}$ Ishiwada, S Echigo, $\mathrm{T}$ Fujiwara, T Murakami, F Ichida, K Joo, N, Haneda, T Higaki, H Suzuki, H Uemura, H Fukushima, and the 66 institutions in Japan for collecting data and, in particular, the assistance of A Niwa MD in preparing the manuscript and analysing the data. We gratefully acknowledge JK Perloff MD and KA Taubert PhD for reviewing the manuscript. This study was supported by grants from the Japanese Society of Paediatric Cardiology and Cardiac Surgery.

\section{Authors' affiliations \\ K Niwa, S Tateno, Chiba Cardiovascular Centre, Chiba, Japan \\ M Nakazawa, Tokyo Women's Medical University, Tokyo, Japan \\ M Yoshinaga, Kagoshima University, Kagoshima, Japan \\ M Terai, Chiba University, Chiba, Japan}

\section{REFERENCES}

1 Dajani AS, Taubert KA, Wilson W, et al. Prevention of bacterial endocarditis: recommendations by the American Heart Association. JAMA. 1997;277: 1794-801, (Circulation, 1997;96:358-66.).

2 Ferrieri $\mathbf{P}$, Gewitz $M H$, Gerber MA, et al. Unique features of infective endocarditis in childhood. Circulation 2002;105:2115-27.

3 Bayer AS, Bolger AF, Taubert KA, et al. Diagnosis and management of infective endocarditis and its complication. Circulation 1998;98:2936-48.

4 Horstkotte D, Follath F, Gutschik E, et al. Guidelines on prevention, diagnosis and treatment of infective endocarditis executive summary. The task force on infective endocarditis of the European Society of Cardiology. Eur Heart J 2004;25:267-76

5 Miyatake K, Akaishi M, Kawasoe K, et al. Guidelines for the prevention and treatment of infective endocarditis (JCS 2003). Circ J 2003;67(suppl IV):1039-110.

6 Niwa K, Nakazawa M, Miyatake K, et al. Survey of prophylaxis and management of infective endocarditis in patients with congenital heart disease: Japanese nationwide survey. Circ J 2003;67:585-91.

7 Perloff JK, Warnes C. Challenges posed by adults with repaired congenital heart disease. Circulation 2001;103:2637-43.

8 Saimon L, Prince A, Gersony WM. Pediatric infective endocarditis in the modern era. J Pediatr 1993;122:847-53.

9 Awadallah SM, Kavey R-EW, Byrum CJ, et al. The changing pattern of infective endocarditis in childhood. Am J Cardiol 1991;68:90-4.

10 Fukushige J, lgarashi H, Ueda K. Spectrum of infective endocarditis during infancy and childhood: 20-year review. Pediatr Cardiol 1994;15:127-31.

11 Li W, Sommerville J. Infective endocarditis in the grown-up congenital heart (GUCH) population. Eur Heart J 1998;19:166-73.

12 Takeda S, Ohta M, Nakazawa M, et al. [Recent experience of infective endocarditis in a single institute: the latest microbiological profile and prevalence of adults with congenital heart disease]. Pediatr Cardiol Card Surg. 2001;17: 534-9, (In Japanese.).

13 Nakatani S, Mitsutake K, Hozumi T, et al. Current characteristics of infective endocarditis in Japan: an analysis of 848 cases in 2000 and 2001. Circ J 2003;67:901-5.

14 Morris CD, Reller MD, Menashe VC. Thirty-year incidence of infective endocarditis after surgery for congenital heart defect. JAMA 1998;279:599-603.

15 Gersony WM, Hayes CJ, Driscoll DJ, et al. Bacterial endocarditis in patients with aortic stenosis, pulmonary stenosis, or ventricular septal defect. Circulation 1993;87(suppl 1):1121-6.

16 Kaplan EL, Rich H, Gersony W, et al. A collaborative study of infective endocarditis in the 1970s: emphasis on infections in patients who have undergone cardiovascular surgery. Circulation 1979;59:327-35.

17 Karl T, Wensley D, Stark J, et al. Infective endocarditis in children with congenital heart disease: comparison of selected features in patients with surgical correction or palliation and those without. Br Heart J 1987;58:57-65.

18 Starke JR. Infective endocarditis. In: Feigin RD, Cherry JD, eds. Textbook of pediatric infectious diseases, 4th ed. Philadelphia: WB Saunders, 1998:315-38.

19 Stanton BF, Baltimore RS, Clemens JD. Changing spectrum of infective endocarditis in children. Am J Dis Child 1984;138:720-5.

20 Durack DT, Kaplan EL, Bisno AL. Apparent failures of endocarditis prophylaxis: analysis of 52 cases submitted to a national registry. JAMA 1983;250:2318-22.

21 Dajani AS, Taubert KA. Infective endocarditis. In: Allen HD, Clark EB, Gutgesell HP, eds. Heart disease in infants, children, and adolescents:including the fetus and young adult, vol II, 6th edn. Baltimore: Williams \& Wilkins, 2001:1297-308

22 Friedman RA, Starke JR. Infective endocarditis. In: Garson A, Bricker JT, McNamara DG, eds. The science and practice of pediatric cardiology. London: Lea \& Febiger, 1990:1561-76.

23 Kavey REW, Frank DM, Byrum CJ, et al. Two-dimensional echocardiographic assessment of infective endocarditis in children. Am J Dis Child 1983; 137:851-6

24 Werner AS, Cobbs CG, Kaye D, et al. Studies on the bacteremia of bacterial endocarditis. JAMA 1967;202:199-203.

25 Bayer AS, Lam K, Ginzton L, et al. Staphylococcus aureus bacteremia: clinical, serologic, and echocardiographic findings in patients with and without endocarditis. Arch Intern Med 1987;147:457-62.

26 Pruitt AA, Rubin RH, Karchmer AW, et al. Neurologic complications of bacterial endocarditis. Medicine 1978;57:329-43.

27 Sanfilippo AJ, Picard MH, Newell JB, et al. Echocardiographic assessment of patients with infective endocarditis: prediction of risk for complications. J Am Coll Cardiol 1991;18:1191-9.

28 Corr $\mathbf{P}$, Wright $M$, Handler LC. Endocarditis-related cerebral aneurysms: radiologic changes with treatment. Am J Neuroradiol 1995;16:745-8.

29 Nomura F, Penny DJ, Menahem S. Surgical intervention for infective endocarditis in infancy and childhood. Ann Thorac Surg 1995;60:90-5.

30 Mosca RS, Bove EL. Surgical therapy of infective endocarditis in children. In: Vlessis AA, Bolling SF, eds. Endocarditis: a multidisciplinary approach to modern treatment. New York: Futura Publishing, 1999:325-37.

31 Reinhartz $O$, Herrmann M, Redling F, et al. Timing of surgery in patients with acute infective endocarditis. J Cardiovasc Surg 1996;37:397-400.

32 Citak M, Rees A, Mavroudis C. Surgical management of infective endocarditis in children. Ann Thorac Surg 1992;54:755-60. 